Letters addressing topics of current interest or material in RESPIRATORY CARE will be considered for publication. The Editors may accept or decline a letter or edit without changing the author's views. The content of letters as published may simply reflect the author's opinion or interpretation of information-not standard practice or the Journal's recommendation. Authors of criticized material will have the opportunity to reply in print. No anonymous letters can be published. Letters should be submitted electronically via Manuscript Central. Log onto RESPIRATORY CARE's web site at http://www.RCJournal.com. Instructions on how to submit a manuscript are on the site and also printed in every issue.

\section{Analysis of Acid-Base Disorders in Patients With Chronic Respiratory Failure}

In a recent paper published in your prestigious journal, Guérin et $\mathrm{al}^{1}$ examined the acid-base disorders in a cohort of patients with chronic respiratory failure. Although I appreciated their contribution of case studies and statistical analysis, I am in partial disagreement with some of their conclusions.

Guérin et al stratified the patients on the basis of stable versus unstable condition and elevated versus not elevated bicarbonate, and examined acid-base balance according to the Stewart approach, the standardized base excess, and the corrected anion gap. In some of the patients they found non-respiratory disorders detected by strong ion difference but not by bicarbonate value, standardized base excess, and corrected anion gap, and they concluded that the diagnostic performance of the Stewart method was better than the conventional approach.

The reliability of Stewart approach, compared to the traditional method to assess acid-base status, is a debated question. ${ }^{2-5}$ As is well known, serum bicarbonate is not an independent variable, and in the traditional approach, which is based on the Henderson-Hasselbalch equation, the acid-base status depends on the ratio of $\mathrm{HCO}_{3}^{-}$to $\mathrm{P}_{\mathrm{CO}_{2}}$, rather than their individual values. $\mathrm{A}$ variation in the bicarbonate level can occur in a primary manner with a metabolic disorder, or can be the consequence of a variation in $\mathrm{P}_{\mathrm{CO}_{2}}$, such as occurs in respiratory disorders. Then, the altered bicarbonate value alone suggests that a patient has an acid-base imbalance, but it is unclear whether the disorder is metabolic or respiratory. Conversely, normal bicarbonate alone does not exclude the presence of a metabolic acid-base imbalance, which could result from the simultaneous presence of acidosis and alkalosis.

The introduction of the standardized base excess, which is used less and less frequently, does not help to clarify this question, because it does not solve the problem of the dependence of bicarbonate on $\mathrm{P}_{\mathrm{CO}_{2}}$, and the use of anion gap is most useful to determine the cause of metabolic acidosis (hyperchloremic rather than normochloremic) once it has been diagnosed.

Certainly, in patients with respiratory failure, respiratory acidosis is the most common acid-base disorder, but metabolic alkalosis and/or acidosis are also frequently observed, and their diagnosis is difficult when a respiratory acidosis coexists.

Respiratory acidosis is characterized by increased $\mathrm{P}_{\mathrm{CO}_{2}}$ and bicarbonate levels. However, the increase in bicarbonate value is greater in the chronic phase than in the acute phase, because renal adaptive changes lead to a raised urinary re-absorption. In the acute phase, the bicarbonate value increases about $1 \mathrm{mEq}$ for every $\mathrm{P}_{\mathrm{CO}_{2}}$ increase of $10 \mathrm{~mm} \mathrm{Hg}$, whereas in the chronic phase the increase is about 3-4 mEq for every $\mathrm{P}_{\mathrm{CO}_{2}}$ increase of $10 \mathrm{~mm} \mathrm{Hg}$. This is the rule of "compensation expected." Consequently, it is important not only to determine if the bicarbonate value is altered, but also the extent of that alteration in relation to changes in arterial $\mathrm{pH}$ and $\mathrm{P}_{\mathrm{CO}_{2}}$.

Surprisingly, in the study by Guérin et al, the mean bicarbonate value was significantly higher in group 3 (unstable patients) than in group 1 (stable patients) (see their Table 4). Excluding an incorrect selection of patients, this finding suggests the presence of a concomitant metabolic alkalosis in many of these patients, whereas the normal bicarbonate in groups 2 and 4 does not exclude the presence of a metabolic disorder. It is therefore not acceptable to reduce the traditional approach to the evaluation only of bicarbonate, standardized base excess, and corrected anion gap.

A stepwise approach has been developed $^{6-8}$ to highlight the presence of a mixed acid-base disorder in the traditional method. Substantial departure from the "compensation expected" suggests a mixed disorder that can be reasonably identified on the basis of discordant variation in $\mathrm{P}_{\mathrm{CO}_{2}}$ and bicarbonate, adequacy of compensatory response, and the delta ratio (ie, the ratio of delta corrected anion gap to delta bicarbonate), as well as anamnesis and physical examination.

Thus, to correctly diagnose a mixed disorder in a patient with respiratory failure according to the traditional approach, all these parameters must be reviewed, and the diagnosis can be made only after assessing them overall. Guérin and colleagues did not provide detailed data on each patient and did not report them to be examined, so I think their conclusion of better performance with the Stewart method is not justified. The traditional approach, with only minor adjustments, can provide the same practical information.

Cosimo Marcello Bruno PhD Department of Internal Medicine and Systemic Diseases University of Catania Catania, Italy

The author has disclosed no conflicts of interest.

\section{REFERENCES}

1. Guérin C, Nesme P, Leray V, Wallet F, Bourdin G, Bayle F, Germain M, Richard JC. Quantitative analysis of acid-base disorders in patients with chronic respiratory failure in stable or unstable respiratory condition. Respir Care 2010;55(11):14531463.

2. Moviat M, van Haren F, van der Hoeven H. Conventional or physicochemical approach in intensive care unit patients with metabolic acidosis. Critical Care 2003;7(3): R41-R45.

3. Sirker AA, Rhodes A, Grounds RM, Bennett ED. Acid-base physiology: the traditional and the modern approaches. Anaesthesia 2002;57(4):348-356.

4. Kurtz I, Kraut J, Ornekian V, Nguyen MK. Acid-base analysis: a critique of the Stewart and bicarbonate-centered approaches. Am J Physiol Renal Physiol 2008;294(5): F1009-F1031.

5. Rastegar A. Clinical utility of Stewart's method in diagnosis and management of acid-base disorders. Clin J Am Soc Nephrol 2009;4(7):1267-1274.

6. DuBose TD Jr. Clinical approach to patients with acid-base disorders. Med Clin North Am 1983;67(4):799-813.

7. Fall PJ. A stepwise approach to acid-base disorders: practical patient evaluation for metabolic acidosis and other conditions. Postgrad Med 2000;107(3):249-250,2534,257-8 passim.

8. Sood P, Paul G, Puri S. Interpretation of arterial blood gas. Indian J Crit Care Med 2010;14(2):57-64. 